\title{
A COMPACT INTEGRATED DEVICE FOR SPATIALLY - SELECTIVE OPTOGENETIC NEURAL STIMULATION BASED ON THE UTAH OPTRODE ARRAY
}

\author{
Robert Scharf $^{* a}$, Christopher F. Reiche ${ }^{* a}$, Niall McAlinden ${ }^{* b}$, Yunzhou Cheng ${ }^{\mathrm{b}}$, Enyuan Xie ${ }^{\mathrm{b}}$, \\ Rohit Sharma ${ }^{a}$, Prashant Tathireddy ${ }^{\mathrm{a}}$, Loren Rieth ${ }^{\mathrm{a}}$, Keith Mathieson ${ }^{\mathrm{b}}$, Steve Blair**a \\ ${ }^{a}$ University of Utah, 50 S. Central Campus Drive, Salt Lake City, UT 84112, USA \\ ${ }^{b}$ University of Strathclyde, TIC, 99 George St, Glasgow G1 1RD, UK \\ * equal contribution **blair@ece.utah.edu
}

\begin{abstract}
Optogenetics is a powerful tool for neural control, but controlled light delivery beyond the superficial structures of the brain remains a challenge. For this, we have developed an optrode array, which can be used for optogenetic stimulation of the deep layers of the cortex. The device consists of a $10 \times 10$ array of penetrating optical waveguides, which are predefined using BOROFLOAT ${ }^{\circledR}$ wafer dicing. A wet etch step is then used to achieve the desired final optrode dimensions, followed by heat treatment to smoothen the edges and the surface. The major challenge that we have addressed is delivering light through individual waveguides in a controlled and efficient fashion. Simply coupling the waveguides in the optrode array to a separately-fabricated $\mu$ LED array leads to low coupling efficiency and significant light scattering in the optrode backplane and crosstalk to adjacent optrodes due to the large mismatch between the $\mu$ LED and waveguide numerical aperture and the working distance between them. We mitigate stray light by reducing the thickness of the glass backplane and adding a silicon interposer layer with optical vias connecting the $\mu$ LEDs to the optrodes. The interposer additionally provides mechanical stability required by very thin backplanes, while restricting the unwanted spread of light. Initial testing of light output from the optrodes confirms intensity levels sufficient for optogenetic neural activation. These results pave the way for future work, which will focus on optimization of light coupling and adding recording electrodes to each optrode shank to create a bidirectional optoelectronic interface.
\end{abstract}

Keywords: optogenetics, waveguide, optrode, microLED, optrode array, microLED array, interposer

\section{INTRODUCTION}

Brain functions emerge through the coordinated activity of neural circuits within and across multiple regions of the cerebral cortex. A central goal in neuroscience is to understand how these circuits generate the cortical computations underlying perception and behavior, which requires perturbing the activity of specific cell types or circuits in a temporally precise manner. Traditionally, this has mainly been performed using electrical stimulation, which, however, lacks spatial precision and cell- or circuit-type specificity. This problem can be overcome by using optogenetics, which allows neural activation or silencing on a millisecond timescale using light. Specific cell types or pathways can be genetically modified to express photosensitive excitatory (e.g. ChR2) or inhibitory (e.g. Arch) opsins[1], [2].

As the model system closest to humans, non-human primates (NHPs) play an important role when studying normal cortical circuit function and the circuit-level basis for human neurological and psychiatric disorders. Being able to perform optogenetic experiments with this animal model is therefore crucial. However, relatively few studies have applied optogenetics in NHPs to this day, which is in part due to the lack of probes for precise light delivery across large brain volumes and through the entire $2 \mathrm{~mm}$ depth of the cortex.

While cortical surface illumination allows for a large coverage area, only penetrating probes give access to deeper tissue (>1 mm), have the necessary spatial precision, and can be modified to also allow electrophysiological recording. Single penetrating optical fibers have already been used[3]-[5], and also been integrated with single[6], [7] and multi-site[8], [9] recording probes, but these are still limited to the illumination of small volumes of tissue. Multisite optical stimulation probes based on fiber optics have also been developed (some also integrated with multisite recording capabilities[10], [11]), but assemblies using high numbers of individually-addressable stimulation sites come with a very invasive cross-section, as shown by a device consisting of four optical fibers surrounding a central recording electrode with a diameter of $\sim 500 \mu \mathrm{m}[12]$. 
Recently, quasi-3D multi-waveguide arrays of varying architectures combining deep-tissue access and increased multisite light delivery have been reported, but, in some cases, the blunt, tapered, polymer optrodes cause problems during insertion and lead to large radiation losses[13], [14]. In other cases, the light transmission efficiency is low[15], [16], or the design makes optical coupling inflexible and challenging[16].

The Utah Electrode Array (UEA) and other kinds of electrode arrays have revolutionized neuroscience by allowing simultaneous recordings from neuronal populations using an innovative, but simple fabrication process. Therefore, the UEA architecture has also been adapted for optical stimulation devices, so-called Utah optrode arrays (UOAs), which make precise optical stimulation of large brain volumes, in acute and chronic studies feasible. One group fabricated a device with $10 \times 10$ square-shaped optrodes, which are up to $1.5 \mathrm{~mm}$-long, and $100 \mu \mathrm{m}$-wide from a transparent conductive oxide substrate ( $\mathrm{ZnO})[17]$, [18]. However, degradation by a local acidic environment associated with the foreign-body response remains an unsolved problem.

We use materials, which have a track record regarding biocompatibility in chronic biomedical implants for our optrode arrays. We build on previous results with fused silica glass[19], [20] and soda-lime glass[21], where light transmittance efficiencies in tissue slices of $90 \%$ throughout the visible spectrum were measured[20]. Our arrays have $10 \times 10$ independent-access sites to reach depths $>1.5 \mathrm{~mm}$, covering a $4 \times 4 \mathrm{~mm}$ tangential area, with cortical surface illumination possible through the backplane of the array. However, the previously-used fused silica was found difficult to structure, and, while giving reasonable results, the resulting optrode arrays were still very rough with sharp edges. In contrast, optrode arrays fabricated from soda-lime glass were found to be relatively easy to structure and could be smoothened with a thermal treatment, but the fabricated optrodes exhibited notable taper along the length of the shank. In the case of the UOAs shown in this publication, we moved to a borosilicate glass (BOROFLOAT® 33, SCHOTT North America, Inc.), which has a coefficient of thermal expansion favorable for integration with silicon structures[22], and was also found to be easy to structure.

The design of the UOAs makes it possible to use broad-area laser sources, which can then simultaneously stimulate large areas on the cortical surface and in the deeper tissue layers[19]. Using careful alignment, individual sites could also be addressed using a focused light source. However, we chose to use $\mu$ LED arrays in order to facilitate light coupling (supported by the flat backplane of the optrode array and by the highly multi-moded nature of optrodes) and to eventually create a fully implantable device. $\mu$ LEDs have recently enabled a new class of thin penetrating probes for deep tissue access (>1 mm) with multiple optical stimulation[23], [24] and optical/electrical recording sites[25], [26] positioned along the axial direction. While tissue heating is the main concern, it has been to shown be manageable and possible to be kept below $0.5^{\circ} \mathrm{C}[23]-[26]$. When the $\mu$ LEDs are positioned outside the tissue, the main advantage of such devices is low tethering forces due to the absence of large fiber bundles and the absence of the need for alignment of an external light source, which would make experiments with freely-behaving animals challenging.

Our $\mu$ LED arrays are fabricated from an InGaN-on-sapphire substrate and contain $181(19 \times 19) \mu L E D s$ in two matrixaddressed arrays: a $10 \times 10$ array to illuminate the optrodes, and an independently-addressable interstitial $9 \times 9$ array for illumination of the cortical surface.

The Lambertian emission profile, as well as a large mismatch in numerical aperture and scattering effects cause optical crosstalk between adjacent optrodes, which makes individual addressing a challenge. We therefore eliminated the glass backplane of the original UEA and instead added a silicon interposer layer with optical vias connecting the $\mu$ LEDs directly to the optrodes. Since the coefficient of thermal expansion of our glass is closely matched to that of silicon, mechanical stability is ensured during the high-temperature fabrication steps.

\section{DEVICE FABRICATION}

\subsection{Overview}

The implantable device for optogenetic stimulation we describe in this publication consist of three main components that are arranged in a stacked fashion: the glass Utah optrode array, the optional optical interposer and the $\mu$ LED array. The optrode array and the $\mu \mathrm{LED}$ array can be fabricated independently from each other and integrated at a later point into a single device. If the optical interposer is required, however, it has to be fabricated prior to the optrode array and then integrated in the optrode fabrication process. 


\subsection{Utah optrode array}

The Utah optrode array fabrication is adapted from previous devices[19], [20] and starts with a 2.3 mm-thick BOROFLOAT ${ }^{\circledR} 33$ glass wafer. The whole process is batch fabrication compatible, meaning that a complete wafer could be processed at once. However, for the optimization of the fabrication parameters, usually smaller sample quantities are required. We therefore cut the glass wafer using a dicing saw (DAD3220, DISCO Corporation) into several pieces of roughly $25 \mathrm{~mm}$ side length, to be able to fabricate smaller batches of 16 arrays. Each piece of substrate is then fixed to a Silicon carrier wafer by using a heat-activated adhesive (WaferGrip, Dynatex International) for increased mechanical stability and better handling during the subsequent steps. For the next step, the dicing saw is used with a bevel blade to cut the surface of the glass substrate in order to create the pyramidal-shaped tips of the optrodes. The facet angle can be adjusted to produce the desired light emission profile. Then, a straight dicing blade is used to define the optrode shanks with a pitch of $400 \mu \mathrm{m}$, a shank edge length of $200 \mu \mathrm{m}$ and height of approximately $1.9 \mathrm{~mm}$, leaving a thin backplane. While this process can be used to create optrode arrays of any dimension, we produced $10 \times 10$ arrays for the device described in this publication. In order to reduce mechanical stress on the dicing blade and the optrodes, this step is performed as a series of four consecutive dicing cuts with increasing depth. By using different dicing parameters and a thicker blade, it is in principle possible to make optrode shanks with even smaller edge lengths. However, we found that optrodes with smaller edge length are more likely to be damaged during the dicing process, consequently reducing the yield of undamaged arrays. The optrode shanks where therefore kept at a diameter of $200 \mu \mathrm{m}$ and are further thinned to their target edge length using wet chemical etching.

As an etchant to thin the optrodes, a hydrofluoric acid solution (concentration of 49\%) is used, buffered with hydrochloric acid solution (concentration of 37\%) at a mixing ration of 9:1. This results in an etch rate of approximately $6 \mu \mathrm{m} / \mathrm{min}$, giving a well-controllable process. After the desired optrode edge length is reached, the optrode is thoroughly rinsed with deionized water and removed from the carrier wafer. In order to remove the residua of the adhesive from the array, a xylenes-based cleaning step is employed[21].

Finally, the cleaned array batch is subjected to a thermal treatment under vacuum in a furnace. The employed heat profile is shown in Figure 1 with the main feature being a high temperature step that keeps the array at $725^{\circ} \mathrm{C}$ for two hours.

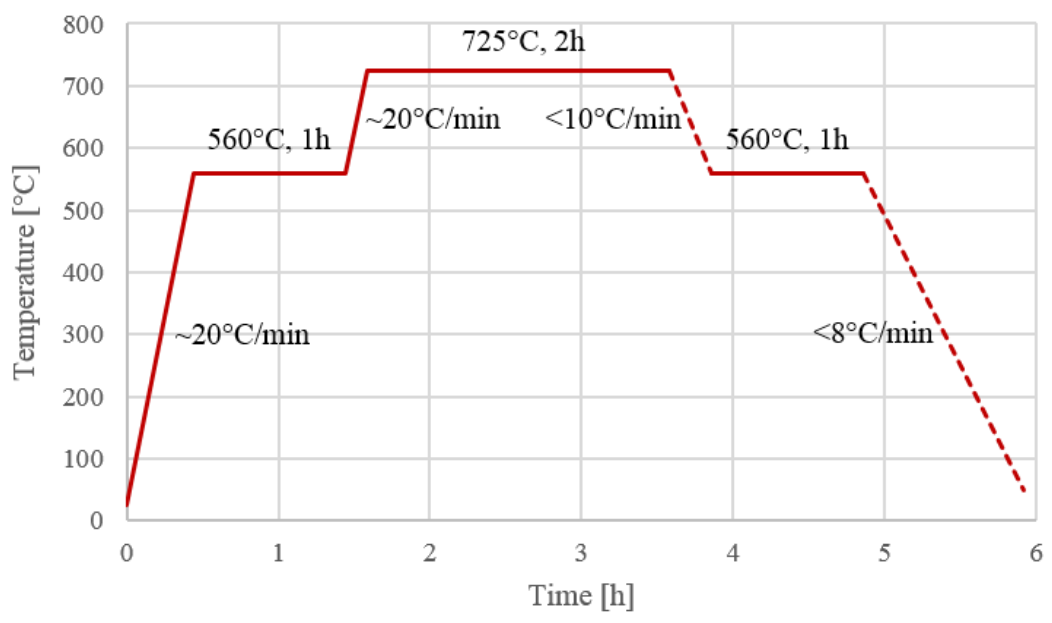

Figure 1: Heat profile of the temperature treatment. The two plateaus at $560^{\circ} \mathrm{C}$ are at the temperature of the annealing point of the glass according to the manufacturer[22]. They are introduced to make sure that the whole glass array is at the same temperature before proceeding and to relief mechanical stress from prior fabrication steps. The plateau parameters at the highest temperature at which the sample is kept at $725^{\circ} \mathrm{C}$ for $2 \mathrm{~h}$ were determined experimentally to give a high level of surface smoothness, while still maintaining the overall shape of the optrodes. Please note that the furnace does not have an active cooling system. Therefore, the rates and actual heat profile of the cooling steps (indicated by the dashed lines) are given as an orientation only and may be lower and look different. 
It was found that this smoothens the roughness on the optrode surface, which exists due to the dicing and etching. It also rounds its features, but without introducing too much distortion of the geometry due to slumping. The noticeable geometry changes include a rounding of the optrodes and the tips as well as a reduction of their length and a slight increase in diameter. After this thermal treatment step, the batch can be singulated into individual arrays using again a carrier wafer with the heat-activated adhesive, the dicing saw, and the final cleaning step. This then results in individual optrode arrays with the desired dimensions. The whole process flow is outlined in Figure 2.

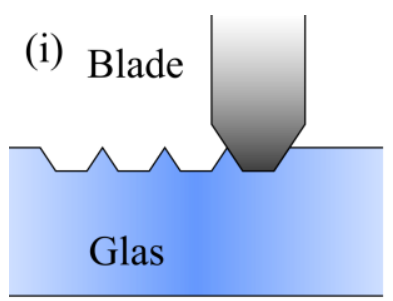

(v)

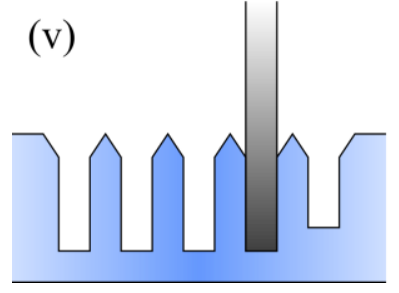

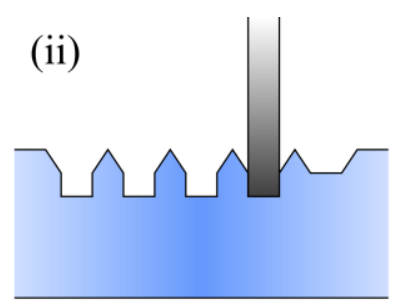

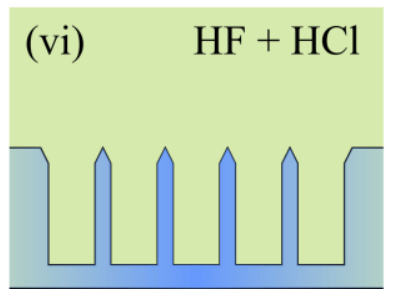

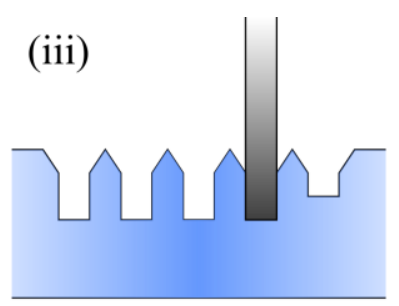

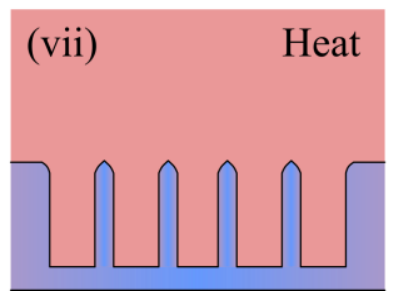

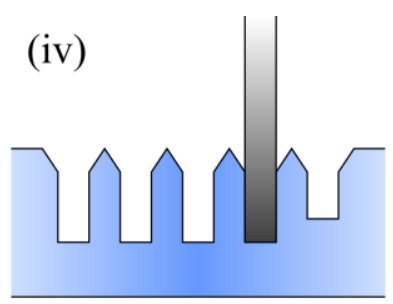

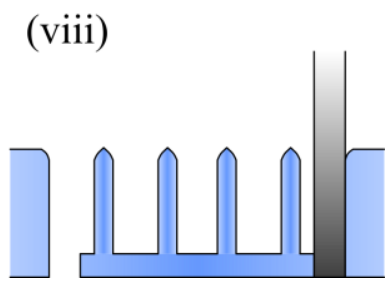

Figure 2: Illustration of the fabrication steps of the Utah optrode array: First, a bevel blade is used to pre-shape the pyramidal optrode tips (i). A straight blade is then employed to cut the optrode shanks in several steps (ii)-(v). To further thin down the optrodes to the target diameter, the array is immersed into an etching solution consisting of hydrofluoric and hydrochloric acid (vi). In order to smoothen the surface and features of the optrodes, they are then subjected to a heat treatment in a furnace (vii). Finally, the arrays are singulated using a straight blade (viii). Please note that the process is illustrated for an array with only 4 optrodes for simplicity. The process is batch compatible and can be adapted for any array dimensions.

\subsection{Optical interposer}

In order to reduce the optical crosstalk between neighboring optrodes, an optical interposer can be added to the backplane of the Utah optrode array, that restricts incoming light in large angles from reaching the optrodes. In our case, the optical interposer is fabricated from a thin $(265 \mu \mathrm{m}$-thick) silicon wafer. The wafer is spin-coated with photoresist (AZ9260, MicroChemicals $\mathrm{GmbH}$ ) and standard photolithography is used to pattern holes corresponding to the required optrode arrangement along with alignment structures in the resist. This pattern is transferred and completely etched thorough the whole wafer using a DRIE process (100 ICP, Oxford). The process cycles between 12 seconds of etching using $\mathrm{SF}_{6}$ and 5 seconds of passivation using $\mathrm{C}_{4} \mathrm{~F}_{8}$ with flow rates of $80 \mathrm{sccm}$ at $15^{\circ} \mathrm{C}$ temperature and $500 \mathrm{~W}$ power. In the next step, the patterned wafer is put onto a $2 \mathrm{~mm}$-thick borosilicate glass wafer suitable for optrode fabrication and is fixed to it using an anodic bonding process at $350^{\circ} \mathrm{C}, 10^{-3} \mathrm{mbar}, 2000 \mathrm{~N}$, and $1000 \mathrm{~V}$ over 20 minutes (520IS, EVG). Afterwards, the glass-interposer assembly is cut into smaller pieces using the dicing saw and the alignment marks in the interposer. The pieces can then be used to fabricate optrode arrays in the glass part using the edges of the sample as alignment marks for the blade cuts to the positions of the interposer holes. Figure 3 shows optrodes on an interposer after dicing and after the finished process. During dicing, $\sim 25 \mu \mathrm{m}$ of glass are left on top of the silicon interposer, which is removed in the wet etch, giving the final optrode dimensions with a support-providing pedestal. 
A

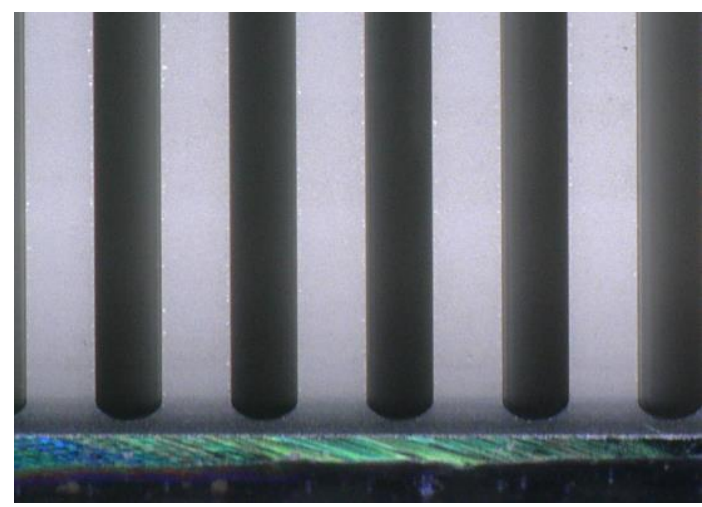

B

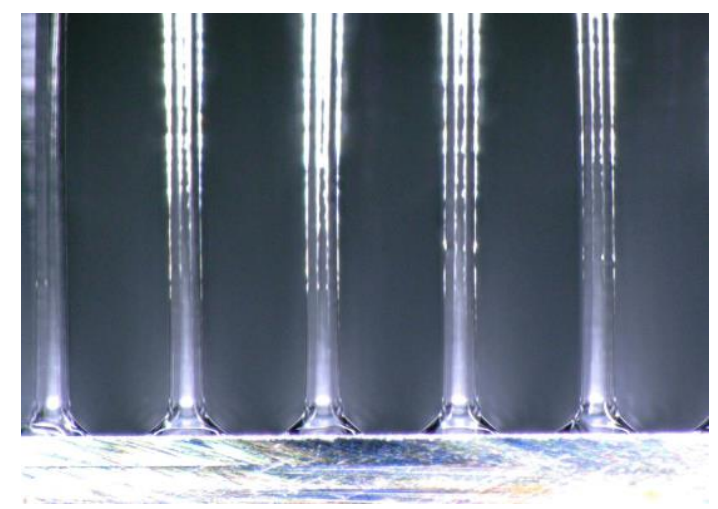

Figure 3: Optrode array on a silicon substrate (interposer), after dicing (A) and after etching and annealing (B). The thin glass backplane that remains after the dicing process is removed in the wet etch, resulting in freestanding optrodes on the silicon interposer, securely held in place over the same-diameter vias by the pedestal that results from the geometry of the dicing blade.

\subsection{LED array}

The $\mu$ LED array is fabricated on a commercial $\operatorname{In}_{x} \mathrm{Ga}_{1-\mathrm{x}} \mathrm{N} / \mathrm{GaN}$ wafer. The typical layer structure of a commercial $\mathrm{In}_{\mathrm{x}} \mathrm{Ga}_{1-\mathrm{x}} \mathrm{N} / \mathrm{GaN}$ wafer is shown in Figure 4. The III-nitride materials are grown on a c-plane (0001) sapphire substrate. As shown, the multi-quantum well (MQW) region includes $\operatorname{In}_{\mathrm{x}} \mathrm{Ga}_{1-\mathrm{x}} \mathrm{N}$ wells and $\mathrm{GaN}$ barriers and is used as an active region for highly radiative, efficient light emission. The MQW region is usually covered by an electron blocking layer (EBL) in a commercial wafer, which is a layer with a higher bandgap energy to prevent the electrons overflowing or escaping from the MQW region. Normally, a p-type wider-bandgap $\mathrm{Al}_{\mathrm{x}} \mathrm{Ga}_{1-\mathrm{x}} \mathrm{N}$ ternary alloy is used as the EBL in the $\mathrm{In}_{\mathrm{x}} \mathrm{Ga}_{1-\mathrm{x}} \mathrm{N} / \mathrm{GaN}$-based LED wafer.

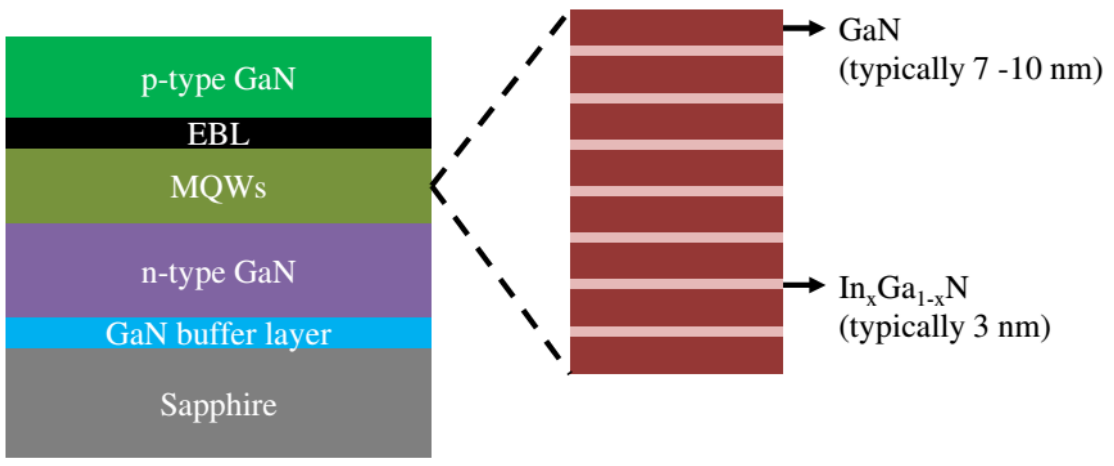

Figure 4: Typical structure of a commercial $\operatorname{In}_{\mathbf{x}} \mathrm{Ga1}$-xN/GaN-based LED wafer grown on a c-plane (0001) sapphire substrate.

The whole fabrication process of the $\mu$ LED array comprises several semiconductor fabrication techniques such as metallization, plasma enhanced chemical vapor deposition (PECVD) and plasma etching. Figure 5 demonstrates the fabrication process flow briefly. 


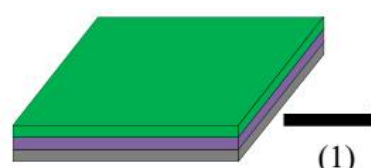

(1)

(3) $\mathrm{Ti} / \mathrm{Au}$ deposition

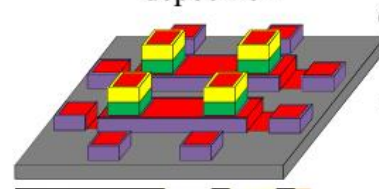

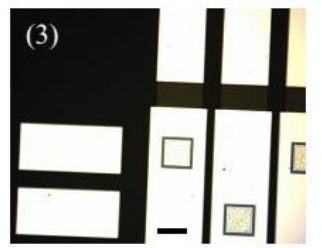

Sapphire $\square$ n-type GaN $\square$ p-type GaN

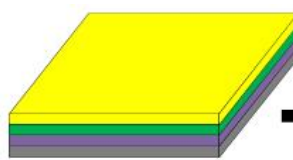

$\mathrm{GaN}$ etching

(2)
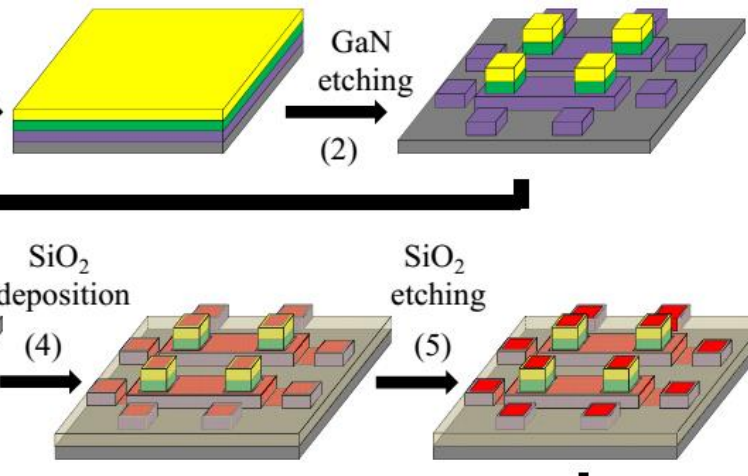

$\mathrm{SiO}_{2}$ etching

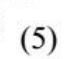

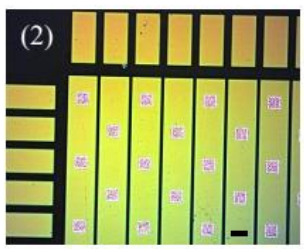
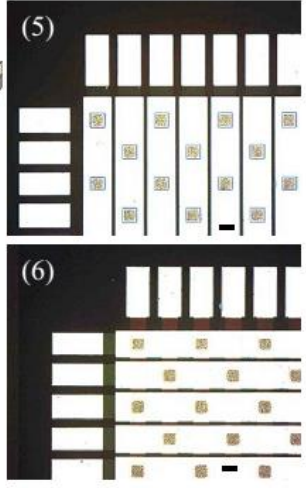

Scale bar: $100 \mu \mathrm{m}$

Figure 5: Fabrication process flow for the $\mu$ LED array. Each image shows the fabrication result of each fabrication step. Scale bars: $100 \mu \mathrm{m}$.

(1) A palladium layer $(100 \mathrm{~nm})$ is firstly deposited onto the wafer as a spreading layer via electron-beam evaporation.

(2) An inductively coupled plasma (ICP) process is used to etch both $\mathrm{p}-\mathrm{GaN}$ and $\mathrm{n}-\mathrm{GaN}$ to define LED pixels and mesa structures. The plasma-forming gases used in the process are argon (Ar) and chlorine $\left(\mathrm{Cl}_{2}\right)$.

(3) A metal layer, that includes titanium $(70 \mathrm{~nm})$ and gold $(300 \mathrm{~nm})$, is sputter deposited to make $\mathrm{n}$-contacts and n-tracks.

(4) A silicon dioxide layer $(300 \mathrm{~nm})$ is deposited via a PECVD process, to form an insulation layer between $\mathrm{n}$-tracks and p-tracks.

(5) The contact regions in both pixels and bonding pads are revealed by a plasma etching process. The plasma-forming gases used in the process are Ar and fluoroform $\left(\mathrm{CHF}_{3}\right)$.

(6) Another metal layer of titanium $(50 \mathrm{~nm})$ and gold $(300 \mathrm{~nm})$ is sputter-deposited to form p-contacts and p-tracks.

\section{MEASUREMENTS}

\subsection{Surface roughness}

Utah optrode arrays, that were made previously from fused silica or soda-lime glass, showed considerable surface roughness when examined using atomic force microscopy[19], [21]. This roughness can lead to light being scattered on the surface along the optrode shank and therefore lost for stimulation. For this reason, we optimized the heat treatment's main parameters, namely the value of the highest applied temperature and the duration it was applied until the optrodes looked optically smooth under the microscope but still maintained their overall shape without bending. The thermal treatment parameters presented in the device fabrication section of this publication are the result of these efforts.

In order to study the surface roughness in more detail, we then used an atomic force microscope (Dimension Icon, Bruker Corporation) in tapping mode operation with a tapping mode cantilever probe (TESPA, Bruker Corporation), measuring the topography on one of the optrode shanks (near the tip).

The result of this measurement is shown in Figure 6. While some larger-scale particle contamination is visible in the scan, the surface roughness in the areas between these larger-scale particles is found to be well below $5 \mathrm{~nm}$, which we believe should not be critical for the intended application. This will, however, be studied further in future publications. The origin of the particle contamination is unknown, but for further studies we will also implement a more thorough cleaning process, especially in the thermal adhesive removal step, as it was found before that this process can create contamination on surfaces. 


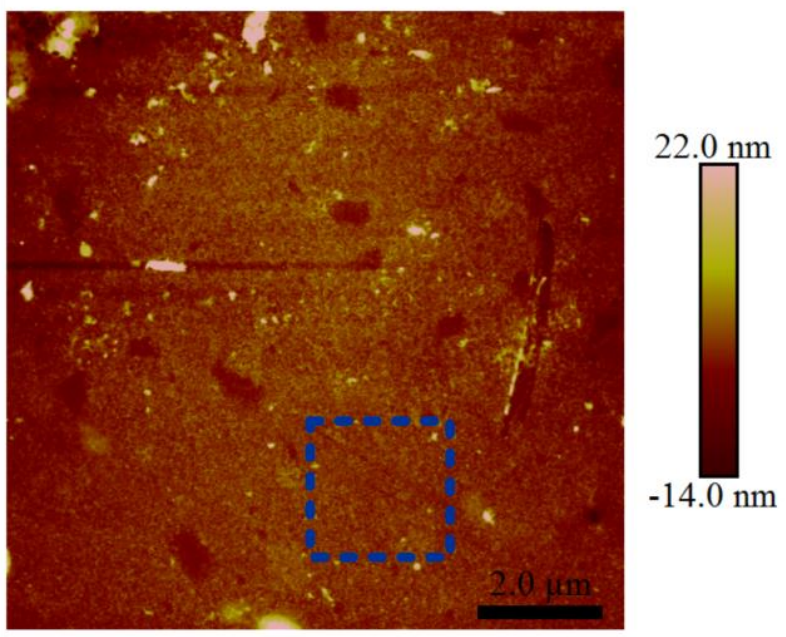

Figure 6: Atomic force microscope topography measurement of the shank surface near the tip of an optrode. The scale was chosen to still sufficiently show the surface roughness between the higher particle contamination. Some of these particles are much larger than the other features and the scale indicates (bright white saturation spots in the image). The box indicates an area where an atomic force microscopy software (NanoScope Analysis 1.50, Bruker Corporation) was used to evaluate the surface roughness between the contamination particles and other surface irregularities. A root-mean-squared roughness of $R_{q}=1.98 \mathrm{~nm}$ was found. A first-order flattening procedure implemented in the software was applied to the data to remove the tilt of the optrode shank with respect to the atomic force microscope.

\subsection{LED performance}

Current-voltage (Figure 7A) and current-light power (Figure 7B) performance of the final device has been measured using a source measure unit (GS610, Yokogawa) and an optical power meter (PM100A, Thorlabs), respectively. A light output of $\sim 5.7 \mathrm{~mW}$ at $120 \mathrm{~mA}$ current corresponds to an Irradiance of $900 \mathrm{~mW} / \mathrm{mm}^{2}$ at the $\mu$ LED surface. The light output from the optrode is significantly lower due to coupling losses. An irradiance of $\sim 1 \mathrm{~mW} / \mathrm{mm}^{2}$ is required for the activation of ChR2.

A

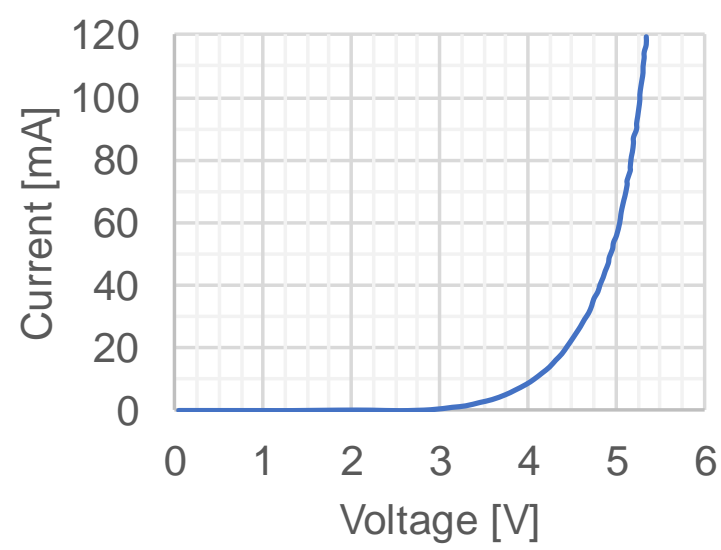

B

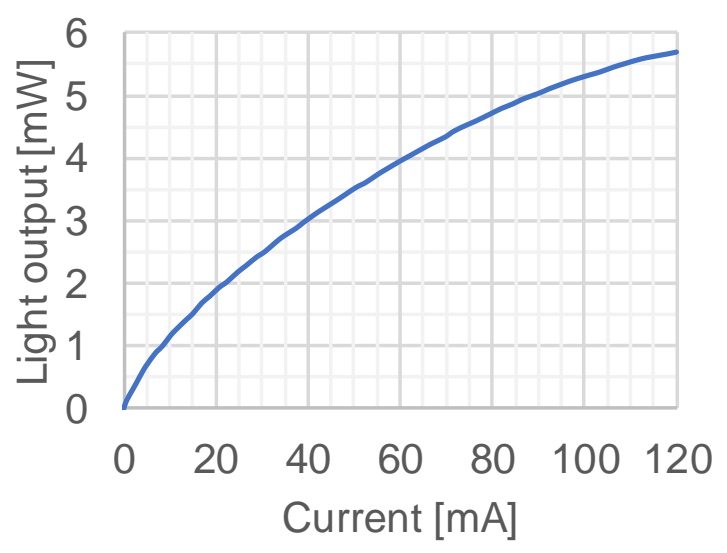

Figure 7: Electrical (A) and optical (B) characterization of a typical $\mu$ LED from the array. 


\subsection{Optical optrode array performance}

Using a photo-diode, that was first placed in front of the $\mu$ LED and then in front of the optrode array aligned on the $\mu$ LED array, a coupling efficiency of $\sim 0.2 \%$ was measured. In order to avoid picking up stray light from the $\mu$ LED substrate (see Figure 8A) during the measurement of the optrode light output, the optrode array was punched through a sheet of aluminum foil, masking everything apart from the optrodes. While the measured efficiency is relatively low, the high brightness of the $\mu$ LEDs (see Figure 7B) still allows irradiances at the optrode tips above the ChR2 activation threshold of $\sim 1 \mathrm{~mW} / \mathrm{mm}^{2}$. The optrodes' emission profile has been assessed by immersing the tips in aqueous fluorescein solution $(10 \mu \mathrm{M}$, see Figure $8 \mathrm{~B})$.

A

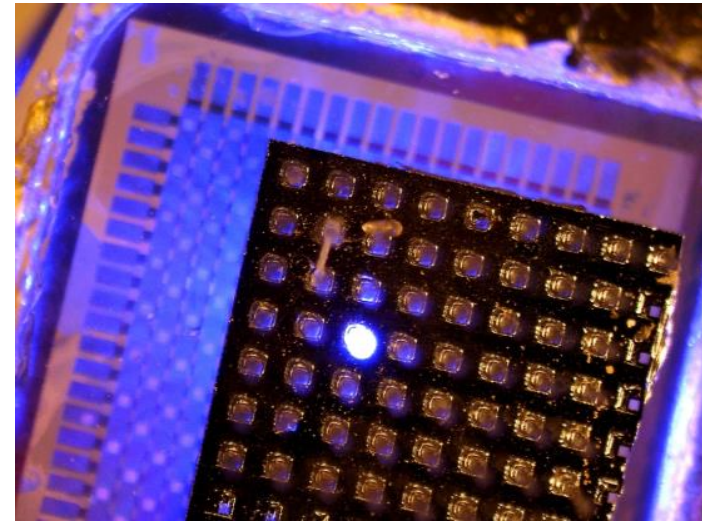

B

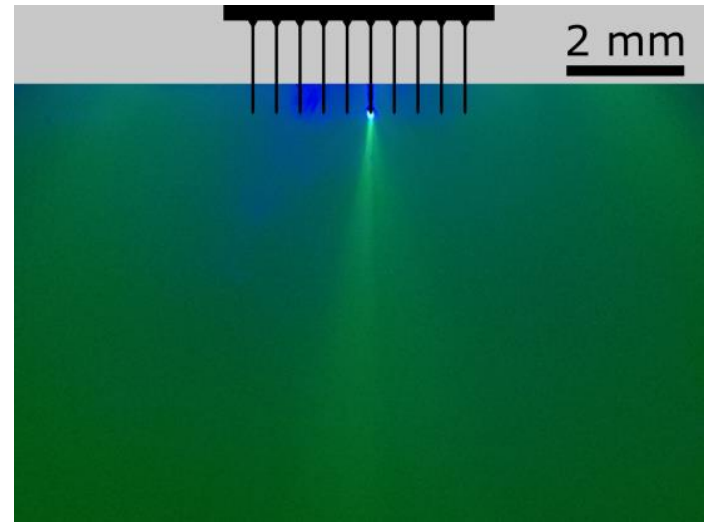

Figure 8: Optrode array coupled to $\mu$ LED array. The interposer restricts the spread of light to one optrode at a time (A). Immersing the array in fluorescein solution shows the emission profile of the optrodes (B).

\section{CONCLUSION}

We have fabricated a Utah optrode arrays from borosilicate glass, that has a coefficient of thermal expansion matched to that of silicon. The fabrication approach - a combination of dicing saw cuts, wet chemical etching and thermal treatment - is highly flexible in terms of array dimensions and is also batch compatible. The resulting optrodes show reasonably low levels of surface roughness. Furthermore, we incorporated an optional optical interposer made from silicon into the fabrication process to reduce optical crosstalk between individual optrodes of the array. To provide illumination through the optrodes, a matching $10 \times 10$ array of $\mu$ LEDs was fabricated based on a commercial $\operatorname{In}_{\mathrm{x}} \mathrm{Ga}_{1-\mathrm{x}} \mathrm{N} / \mathrm{GaN}$ wafer. The reduced surface roughness of the optrodes as compared to previous approaches, as well as the performance of the $\mu$ LEDs and the coupled optrode array-interposer- $\mu$ LED structure was verified experimentally. It was found that a fully integrated device should be able to reach irradiances at the optrode tips above the ChR2 activation threshold for optogenetic stimulation.

We are going to permanently bond the $\mu$ LED array to the optrode array, which will then allow us to do acute and chronic optogenetic studies in the NHP and other large brains (using one or more arrays implanted in parallel). Our technology is applicable to the chronic behaving monkey preparation, thus making it feasible to study neural circuit function in an animal species capable of complex behavior. We will furthermore implement the electrical recording capabilities of the Utah electrode array, allowing us to record from large neuronal populations while stimulating with spatiotemporal patterns that mimic neural activity during natural perception or behavior.

\section{REFERENCES}

[1] L. Luo, E. M. Callaway, and K. Svoboda, "Genetic dissection of neural circuits," Neuron, vol. 57, no. 5, pp. 634660, Mar. 2008.

[2] F. Zhang, A. M. Aravanis, A. Adamantidis, L. de Lecea, and K. Deisseroth, "Circuit-breakers: optical technologies for probing neural signals and systems," Nat. Rev. Neurosci., vol. 8, no. 8, pp. 577-581, Aug. 2007.

[3] J. Cavanaugh et al., "Optogenetic inactivation modifies monkey visuomotor behavior," Neuron, vol. 76, no. 5, pp. 901-907, Dec. 2012. 
[4] A. Gerits, R. Farivar, B. R. Rosen, L. L. Wald, E. S. Boyden, and W. Vanduffel, "Optogenetically-induced behavioral and functional network changes in primates," Curr. Biol. CB, vol. 22, no. 18, pp. 1722-1726, Sep. 2012.

[5] X. Han et al., "Millisecond-timescale optical control of neural dynamics in the nonhuman primate brain," Neuron, vol. 62, no. 2, pp. 191-198, Apr. 2009.

[6] V. Gradinaru et al., "Targeting and Readout Strategies for Fast Optical Neural Control In Vitro and In Vivo," J. Neurosci., vol. 27, no. 52, pp. 14231-14238, Dec. 2007.

[7] I. Ozden et al., "A Coaxial Optrode As Multifunction Write-Read Probe for Optogenetic Studies in Non-Human Primates," J. Neurosci. Methods, vol. 219, no. 1, pp. 142-154, Sep. 2013.

[8] P. Anikeeva et al., "Optetrode: a multichannel readout for optogenetic control in freely moving mice," Nat. Neurosci., vol. 15, no. 1, pp. 163-170, Dec. 2011.

[9] A. V. Kravitz et al., "Regulation of parkinsonian motor behaviors by optogenetic control of basal ganglia circuitry," Nature, vol. 466, no. 7306, pp. 622-626, Jul. 2010.

[10] S. Royer, B. V. Zemelman, M. Barbic, A. Losonczy, G. Buzsáki, and J. C. Magee, "Multi-array silicon probes with integrated optical fibers: light-assisted perturbation and recording of local neural circuits in the behaving animal," Eur. J. Neurosci., vol. 31, no. 12, pp. 2279-2291, Jun. 2010.

[11] E. Stark, T. Koos, and G. Buzsáki, "Diode probes for spatiotemporal optical control of multiple neurons in freely moving animals," J. Neurophysiol., vol. 108, no. 1, pp. 349-363, Jul. 2012.

[12] K. Tamura et al., "A glass-coated tungsten microelectrode enclosing optical fibers for optogenetic exploration in primate deep brain structures," J. Neurosci. Methods, vol. 211, no. 1, pp. 49-57, Oct. 2012.

[13] K. Y. Kwon, A. Khomenko, M. Haq, and W. Li, "Integrated slanted microneedle-LED array for optogenetics," Conf. Proc. Annu. Int. Conf. IEEE Eng. Med. Biol. Soc. IEEE Eng. Med. Biol. Soc. Annu. Conf., vol. 2013, pp. 249$252,2013$.

[14] K. Kwon and W. Li, "Integrated multi-LED array with three-dimensional polymer waveguide for optogenetics," in 2013 IEEE 26th International Conference on Micro Electro Mechanical Systems (MEMS), 2013, pp. 1017-1020.

[15] F. Wu et al., "An implantable neural probe with monolithically integrated dielectric waveguide and recording electrodes for optogenetics applications," J. Neural Eng., vol. 10, no. 5, p. 056012 , Oct. 2013.

[16] A. N. Zorzos, E. S. Boyden, and C. G. Fonstad, "Multiwaveguide implantable probe for light delivery to sets of distributed brain targets," Opt. Lett., vol. 35, no. 24, pp. 4133-4135, Dec. 2010.

[17] J. Lee, Y. K. Song, I. Ozden, and A. V. Nurmikko, "Transparent micro-optrode arrays for simultaneous multichannel optical stimulation and electrical recording," in CLEO: 2013, 2013, pp. 1-2.

[18] J. Lee, I. Ozden, Y.-K. Song, and A. V. Nurmikko, "Transparent intracortical microprobe array for simultaneous spatiotemporal optical stimulation and multichannel electrical recording," Nat. Methods, vol. 12, no. 12, pp. 11571162, Dec. 2015.

[19] T. V. F. Abaya, S. Blair, P. Tathireddy, L. Rieth, and F. Solzbacher, "A 3D glass optrode array for optical neural stimulation," Biomed. Opt. Express, vol. 3, no. 12, pp. 3087-3104, Dec. 2012.

[20] T. V. F. Abaya, M. Diwekar, S. Blair, P. Tathireddy, L. Rieth, and F. Solzbacher, "Deep-tissue light delivery via optrode arrays," J. Biomed. Opt., vol. 19, no. 1, p. 015006, Jan. 2014.

[21] R. W. Boutte and S. Blair, "Maskless wafer-level microfabrication of optical penetrating neural arrays out of sodalime glass: Utah Optrode Array," Biomed. Microdevices, vol. 18, no. 6, p. 115, Dec. 2016.

[22] SCHOTT North America, Inc., "BOROFLOAT® 33 - Thermal Properties." [Online]. Available: http://www.us.schott.com/borofloat/english/download/index.html. [Accessed: 08-Jan-2018].

[23] N. McAlinden et al., "Thermal and optical characterization of micro-LED probes for in vivo optogenetic neural stimulation," Opt. Lett., vol. 38, no. 6, pp. 992-994, Mar. 2013.

[24] R. Scharf, T. Tsunematsu, N. McAlinden, M. D. Dawson, S. Sakata, and K. Mathieson, "Depth-specific optogenetic control in vivo with a scalable, high-density $\mu$ LED neural probe," Sci. Rep., vol. 6, p. srep28381, Jun. 2016.

[25] T. Kim et al., "Injectable, cellular-scale optoelectronics with applications for wireless optogenetics," Science, vol. 340, no. 6129, pp. 211-216, Apr. 2013.

[26] F. Wu, E. Stark, P.-C. Ku, K. D. Wise, G. Buzsáki, and E. Yoon, "Monolithically Integrated $\mu$ LEDs on Silicon Neural Probes for High-Resolution Optogenetic Studies in Behaving Animals," Neuron, vol. 88, no. 6, pp. 11361148, Dec. 2015. 